\title{
Kunstwerke im Palmengarten - Schenkungen von Bankiersfamilien und schöne Frauen
}

Herbert Billensteiner \& Hilke Steinecke

\section{Schenkungen der Bethmanns}

Vor dem Haus Leonhardsbrunn ruhen jeweils auf einem Podest zwei überlebensgroße steinerne Löwen. Die aus rotem Sandstein gearbeiteten Sphinx-ähnlichen Figuren standen früher vor dem „Ariadneum“ im Bethmannpark. Sie waren mehr als 100 Jahre im Besitz der Familie von Bethmann und wurden nach Abriss des Hauses von der Familie am 15. 3. 1961 dem Palmengarten gestiftet. Sie wurden zuerst in der Nähe des Kinderspielplatzes beim großen Weiher aufgestellt. Familie Bethmann ist eine alteingesessene Frankfurter Kaufmanns- und Bankiersfamilie. Ihr Bankhaus wurde im Jahre 1748 gegründet. Das europäische Anleihegeschäft florierte, bis Anfang des 19. Jh. auf diesem Sektor die Rothschilds marktführend wurden. Bethmanns verlagerten daraufhin ihre Schwerpunkte und engagierten sich z. B. intensiv im Bau von Eisenbahnstrecken. So ist es doch sehr passend, dass die Löwen in der Nähe der Haltestelle des Palmen-Express platziert sind.

\section{Schenkungen der RothsCHILDs}

Auch die jüdische Bankiersfamilie Rothschild hat ihr Stammhaus in Frankfurt. Familienzweige reichen nach England, wo 1885 dem Londoner Bankier Nathan Mayer Rothschild (1840-1915) erstmals der erbliche Adelstitel Baron Rothschild verliehen wurde. Freifrau Louise von Rothschild (1820-1894), eine Enkelin von Mayer Amschel Rothschild (1744-1812), dem Begründer der RothschildDynastie, lebte in Frankfurt. Zusammen mit ihrem Gatten Mayer Carl von Rothschild engagierte sie sich sehr für Frankfurt und machte verschiedene Stiftungen. Darunter war auch das Clementine-Krankenhaus, das sie in Erinnerung an ihre jung verstorbene Tochter Clementine bauen ließ. Dem Palmengarten stiftete sie 1892, also kurz vor ihrem Tod, fünf um 1830 geschaffene Marmorfiguren (vier Jahreszeiten-Figuren, Pluto) und die im Rosengarten auf Sockeln stehenden Marmorvasen.

Die Figur des Pluto wurde zuerst gegenüber dem Eingang an der Westseite des Gesellschaftshauses aufgestellt. Heute steht der aus der griechischen Mythologie stammende Gott Pluto neben dem Magnolienhain auf der Wiese gegenüber der Villa Leonhardi. Pluto wurde als Gott der aus der Erde kommenden Reichtümer betrachtet, wachte also auch über Getreidevorräte und keimende Pflanzen. Später wurde er der Herrscher über das Totenreich und die Unterwelt und ist somit HADES gleichzusetzen. Nicht zu übersehen sind die Totenköpfe am Fuße der Pluto-Figur.

Drei der vier 1,80 m hohen Marmor-Frauen wurden 1989 von der Steinmetzfirma KoHLMANN aus Bockenheim an der Westseite des nunmehr dritten Rosengartens aufgestellt. Herbst und Sommer befinden sich heute unter der Pergola. Die Frühlingsfigur ist etwas versteckt in einer Gehölzgruppe zwischen Rosengarten und Liegewiese untergebracht. Die Figur des Winters ist verschollen. Leider ist über den Künstler nichts bekannt.

\section{Stille Frauen des Palmengartens}

Neben den drei Jahreszeiten-Figuren beherbergt der Palmengarten noch einige weitere weibliche Figuren, die stumme Zeugen der Geschichte des Palmengartens sind.

Georg Hartmann, Ehrenbürger der Stadt Frankfurt, schenkte der Stadt Frankfurt die Bronzefigur „FlorA“, eine knieende Frau mit vorgestreckten Händen. Sie ist etwa $130 \mathrm{~cm}$

Abb. 1 (Seite 67 oben): Einer der beiden Löwen vor dem Haus Leonhardsbrunn.

Abb. 2 (Seite 67 unten): Historische Ansichtskarte Blick in die alte Mittelhalle mit Marmorvasen. 

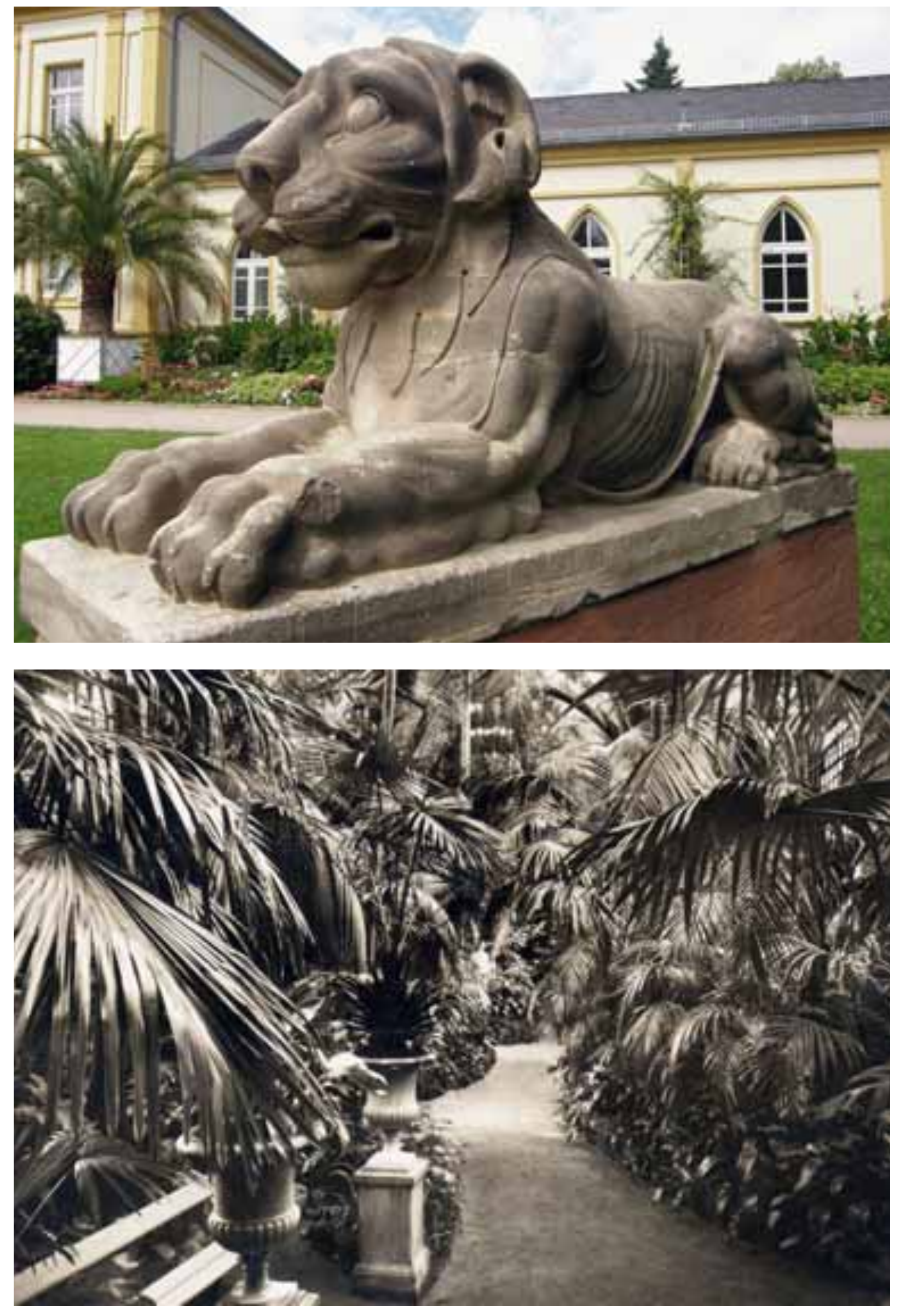

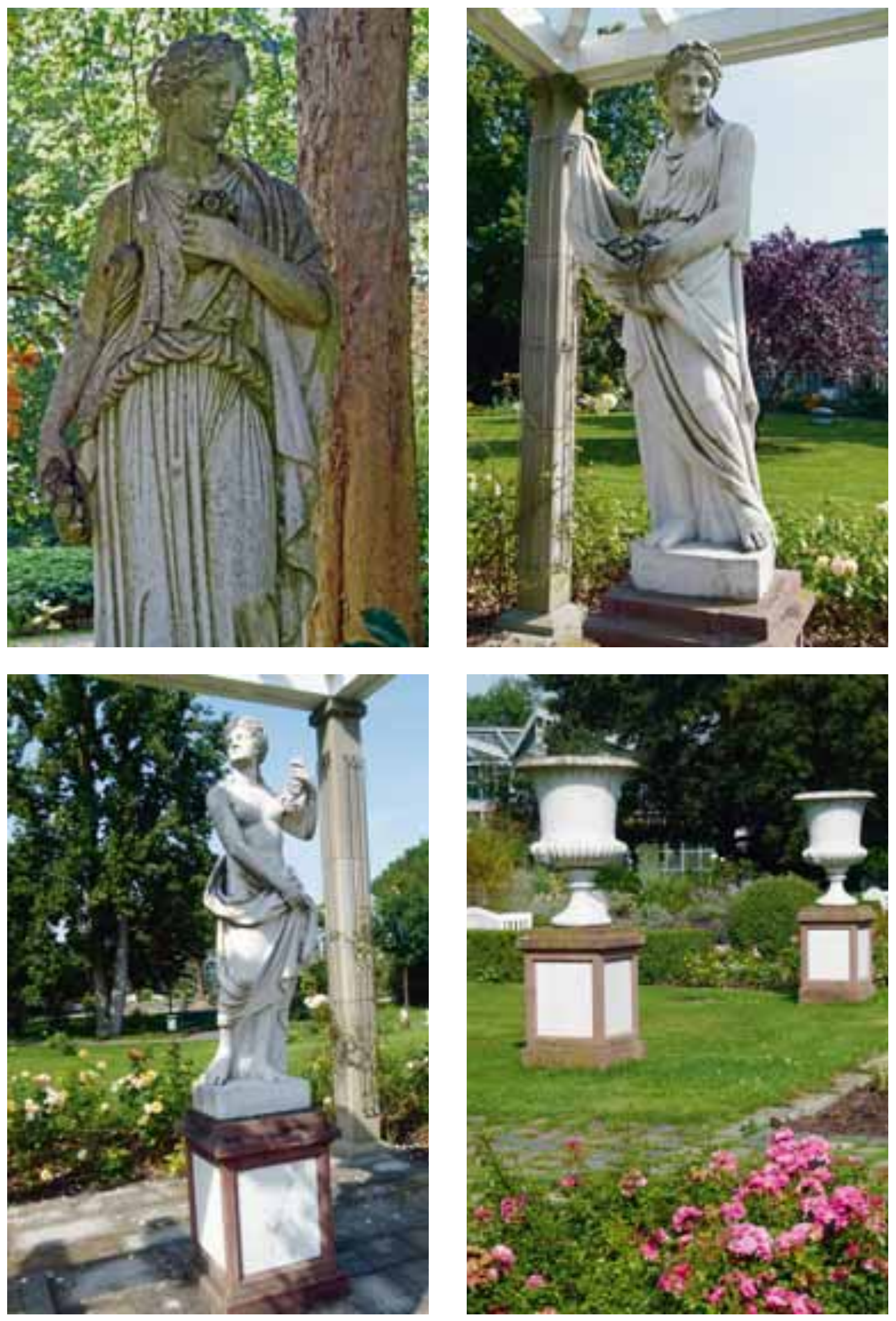
hoch und wurde im Juni 1952 im Palmengarten aufgestellt. Geschaffen hat sie um 1930 der Bildhauer Richard Petraschke, der am 26.3. 1885 in Schaffhausen geboren wurde und 1904 nach Frankfurt kam. Dort gründete er 1913 sein eigenes Atelier. Es heißt, dass die Kunst und die Arbeit seine Leidenschaft gewesen seien. Im Frankfurt der 1930er Jahre gehörte Petraschke zu den führenden Plastikern. Er starb am 19. 11. 1937 in Frankfurt.

Frau Braun-Marschner stiftete 1972 die $156 \mathrm{~cm}$ hohe Bronzefigur „Die Tänzerin“. Geschaffen wurde sie von Arthur LEwiNFuncke im Jahre 1903. Der Künstler wurde am 9. 11. 1866 in Niedersedlitz bei Dresden geboren. Nach dem Studium gründete er ein eigenes Atelier. Im Jahre 1916 schuf er eine ähnliche Figur „Hingebung“. Sie steht heute im Botanischen Garten und Museum Berlin. LewinFunCKE starb am 16. 10. 1937 in Berlin.

Von der Stadt Frankfurt in Auftrag gegeben wurde die „Märchenerzählerin“ Elisabeth Textor-Goethe mit Sohn Wolfgang, eine $140 \mathrm{~cm}$ hohe Plastik. Zusammen mit dem Muschelkalksockel ist sie $2 \mathrm{~m}$ hoch. Der Künstler Josef Kowarzik wurde am 1. 3. 1860 in Wien geboren. Im Jahre 1897 kam er nach Frankfurt, wo er an der Kunstgewerbeschule und am Städel'schen Institut lehrte. Von ihm stammt auch ein Reliefbild aus Bronze mit ähnlicher Darstellung von 1908. Der Künstler starb am 13. 3. 1911 in Frankfurt. Die Figur vollendete Bernhard Hoetgen (1874-1949). Sie wurde 1915 im Palmengarten aufgestellt. Seit 1999 steht sie am jetzigen Standort, wo sich nun auch der Goethe-Garten befindet.

Herr F. Schumacher schenkte am 9. 10. 1982 dem Palmengarten für das Palmenhaus

Abb. 3 (Seite 68 oben links): Frühlings-Figur. Abb. 4 (Seite 68 oben rechts): Sommer-Figur. Abb. 5 (Seite 68 unten links): Herbst-Figur. Abb. 6 (Seite 68 unten rechts): Marmorvasen im Rosengarten.

Abb. 7 (oben): "Pluto"

Abb. 8 (unten): "FlorA"
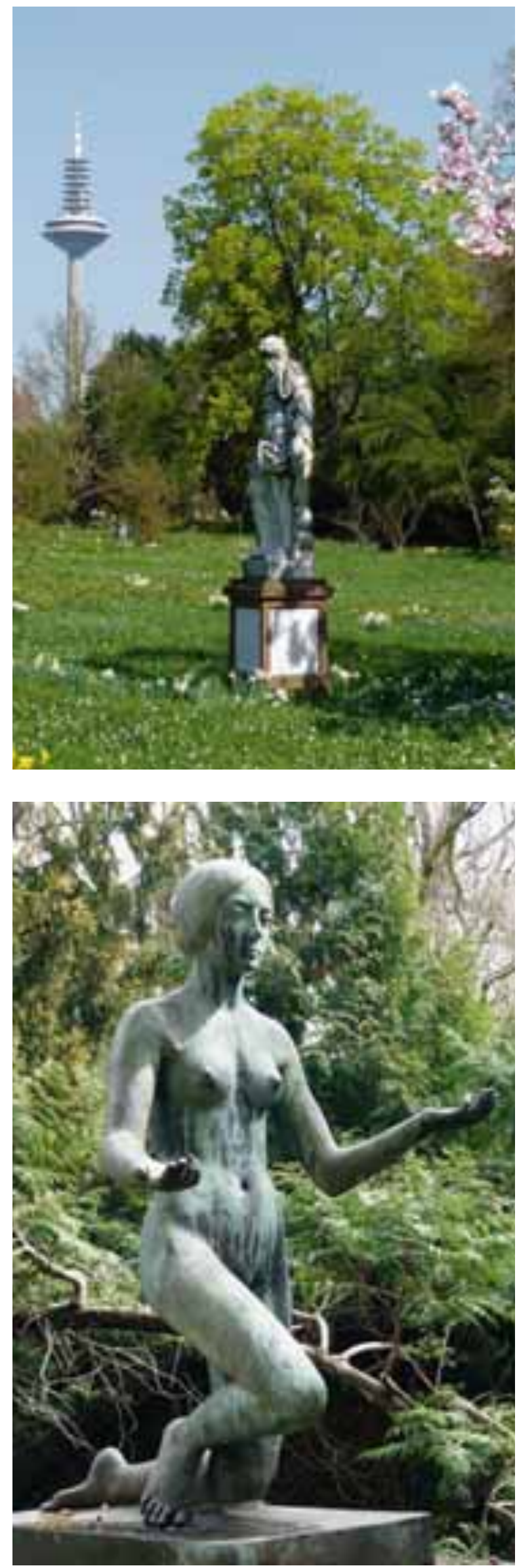

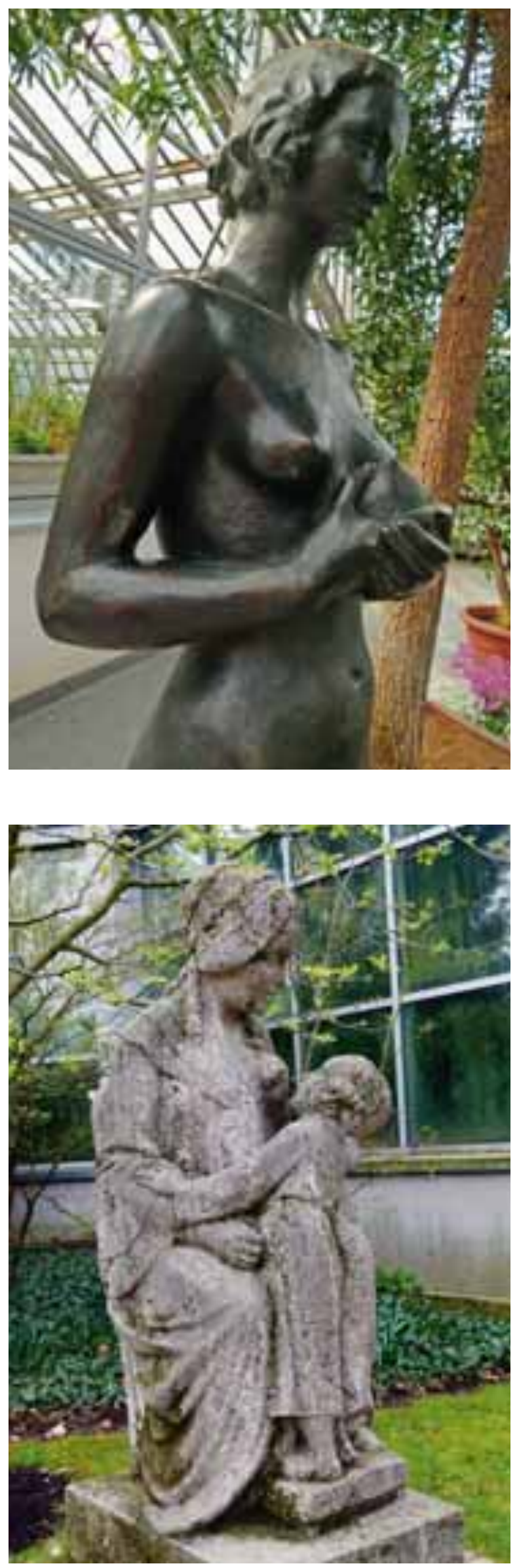

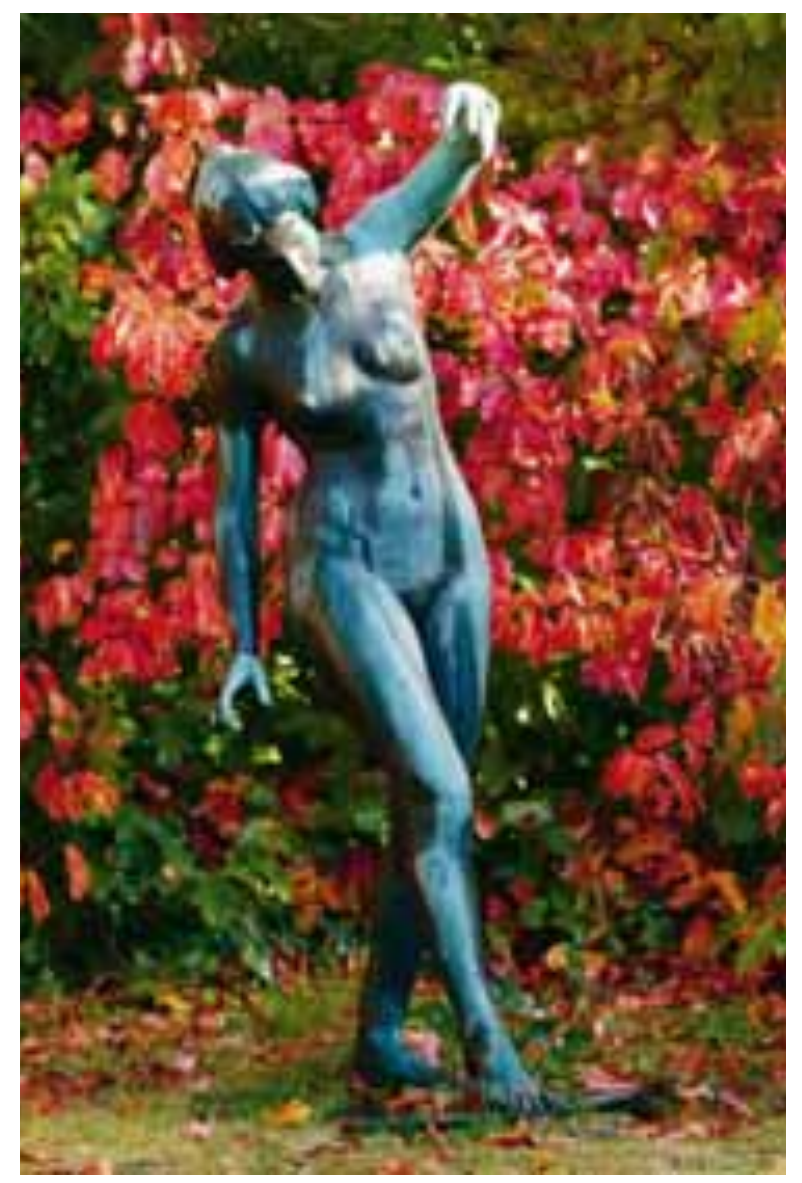

die $146 \mathrm{~cm}$ hohe Bronzefigur „EvA“. Es handelt sich um das Original von drei Abgüssen des Bildhauers Fritz KlimsCh, der am 10. 2. 1870 in Frankfurt (Oberlindau 15) geboren wurde. Im Jahr 1886 besuchte er die Königliche Akademische Hochschule der Bildenden Künste Berlin. Ab 1921 war er Professor an der Akademie der Bildenden Künste Berlin, 1925 wurde "Eva" in der Gießerei Noack in Berlin gegossen. Der Reichsminister Joseph Goebbels kaufte „EvA“. Auch sein Ministerium für Volksaufklärung und Propaganda ließ er von KLIMSCH verschönern. Die Familie KLIMSCH kaufte später die Figur zurück. Der Künstler starb am 30. 3. 1960 in Freiburg. „EvA“ steht zur Zeit im historischen Eingangsschauhaus des Palmengartens.

Abb. 9 (oben links): "Eva"

Abb. 10 (oben rechts): "Tänzerin" im Herbst vor dem leuchtend rot gefärbten Eisenholzbaum (Parrotia persica).

Abb. 11 (unten): "Märchenerzählerin". 